\title{
Biometric identification using local iterated function
}

\begin{abstract}
Biometric identification protocol has been received an increasing interest recently. It is a process that determines person identity by making use of their biometric features. A new biometric identification method is presented in this paper based on partial self-similarity that used to identify features within fingerprint images. This approach is already used in Fractal Image Compression (FIC) due to their ability to represent the images by a limited number of affine transformations, and its variation of scale, translation or rotation. These features give the recognition process high impact and good performance. To process data in a fingerprint image, it first converted into digital format using Optical Fingerprint Reader (OFR). The verification process is done by comparing these data with the server data. The system analysis shows that the proposed method is efficient in terms of memory and time complexity.
\end{abstract}

Keyword: Biometric identification protocol; Iterated function systems (IFS); Optical fingerprint reader (OFR); Fingerprint 